\title{
Des discours pour condamner un usage dévoyé du langage : une analyse des discours autour du prix "Orwell Novlang » des Big Brother Awards
}

\author{
Krieg-Planque, Alice \\ Université Paris-Est Créteil (UPEC) - Céditec (EA 3119) \\ krieg-planque@u-pec.fr
}

\begin{abstract}
Dans cette contribution, nous proposons d'analyser des discours qui sont produits par un collectif militant (en l'occurrence la section française de l'association Privacy International) dans le cadre de la remise d'un prix qui concerne l'usage de la langue et/ou du discours (à savoir le prix « Orwell Novlang » des Big Brother Awards). C'est donc la fonction métalinguistique du langage qui nous occupe ici, telle que la mettent en œuvre des locuteurs « ordinaires » (entendus ici comme des non-spécialistes du savoir savant sur la langue et sur le discours) et telle qu'elle participe d'une démarche de critique politique. Au moyen de cette étude, nous espérons contribuer, depuis le champ de l'analyse du discours, aux réflexions sur le métalangage ordinaire, spécialement dans ses usages critiques, militants et/ou polémiques en contexte socio-politique.

A travers l'analyse de textes rédigés par les militants organisateurs des Big Brother Awards, nous cherchons à saisir les appréciations et les jugements que ces locuteurs portent sur l'emploi du langage, en particulier lorsqu'ils renvoient ces emplois à ce qu'ils appellent eux-mêmes une ou la «novlangue ». En effet, nous partons du principe que la «novlangue » n'existe pas en elle-même, de manière naturelle ou objectivable, mais plutôt comme un terme métalinguistique ordinaire qui permet de référer, pour des locuteurs engagés dans une démarche critique, à un ensemble relativement diffus d'usages. Ce sont ces usages que nous cherchons à identifier, par l'analyse des textes des argumentaires produits par l'association pour instruire les dossiers des nominés (soit 40 argumentaires étudiés de 2004 à 2013).
\end{abstract}

\section{Le contexte de l'étude : un anti-prix comme démarche militante visant des pratiques langagières}

\subsection{Les Big Brother Awards : un collectif producteur de discours critiques}

De manière à situer les discours étudiés, et avant d'aborder la dimension strictement langagière de notre sujet, nous présentons de façon synthétique le collectif militant qui en est le producteur. Nous nous appuyons pour cela sur la documentation qui est mise en ligne sur le site web de Privacy France, ainsi que sur l'ouvrage publié par les Big Brother Awards (2008) pour faire le bilan de leur propre activité. A cette documentation écrite s'ajoute notre observation de trois événements organisés par Privacy France : en effet, notre intérêt personnel pour la thématique des droits humains en rapport avec l'usage des données personnelles nous a amenée à assister, comme simple participante dans le public, aux éditons 2004, 2007 et 2013 de la cérémonie de remise des Big Brother Awards.

L'association Privacy France est la section française de Privacy International, association basée à Londres et qui se donne pour mission de défendre le droit à la vie privée (surveillance des citoyens par les Etats, contrôle illégal des salariés par leurs employeurs, détournements de listes électorales ou de fichiers de clientèle...). Animée par une quinzaine de militants actifs, Privacy France est engagée en faveur de cette même cause, mais elle concentre son énergie sur un instrument, les Big Brother Awards : l'organisation de cette cérémonie de remise de prix est en effet l'action principale de ce petit collectif.

Les Big Brother Awards sont présentés par Privacy France comme les « césars du monde sécuritaire ». Toujours suivant les termes de l'association, de tels « césars» visent à « récompenser les acteurs de la société de contrôle et de surveillance », quels qu'ils soient : selon la précision donnée par le collectif, 
« toute institution, entreprise ou personne s'étant distinguée par son mépris du droit à la vie privée et/ou par sa promotion de la surveillance et du contrôle des individus peut être suggérée comme candidate. » Comme on le comprend, les récompenses décernées ne sont donc pas tout à fait des " prix » mais plutôt des « anti-prix » : elles ont pour but de dénoncer des pratiques. A ces anti-prix s'ajoute un véritable prix, le «Prix Voltaire », qui distingue un acteur positif de la défense du droit à la vie privée.

Le principe d'organisation des Big Brother Awards est le suivant. Pendant les mois qui précèdent la remise des prix, les membres du collectif, et leurs sympathisants sollicités par une newsletter, mènent une activité de veille documentaire pour repérer des candidats potentiels. Ayant vérifié les faits incriminés, et sourcé précisément les informations, les organisateurs sélectionnent des nominés (environ 4 à 8 par catégorie de prix) pour chacun desquels ils rédigent un argumentaire, qui constitue le dossier à charge du candidat. L'ensemble des argumentaires est présenté à un jury composé d'une dizaine de personnalités, choisies pour leur attachement au droit comme levier d'action (membres de la Ligue des droits de l'homme, du Syndicat de la magistrature, du Syndicat des avocats de France...), ou encore pour leurs compétences critiques en matière de théorie politique des réseaux et de l'internet (Benjamin Bayart, Xavier de La Porte, Jean-Marc Manach, Jérôme Thorel, Jérémie Zimermann ...). Le jury ayant délibéré, l'annonce des lauréats est proclamée publiquement lors de la cérémonie de remise des Big Brother Awards.

Cette cérémonie se déroule dans un lieu public, à Paris ou en proche banlieue parisienne, dans des locaux à coloration alternative mais habilités à recevoir du public. Par exemple, environ 200 personnes étaient rassemblées à La Parole Errante (à Montreuil, en Seine-Saint-Denis) pour la onzième édition des Big Brother Awards qui s'est déroulée au cours d'une soirée d'un peu plus de 3 heures, le mercredi 26 juin 2013. Lors de la cérémonie, les organisateurs résument à l'attention du public les argumentaires des nominés, et donnent lecture des argumentaires des lauréats de chacun des prix.

La démarche adoptée par le collectif des Big Brother Awards est réellement une démarche militante : il s'agit de faire valoir une cause, de défendre un point de vue, d'apporter à la discussion des éléments de critique auxquels les militants espèrent sensibiliser le grand public. Les organisateurs présentent d'ailleurs explicitement les Big Brother Awards comme relevant d'une démarche de name and shame, terme anglais appartenant au lexique de la sociologie des mouvements sociaux et qui désigne les pratiques de dénonciation et de mise en visibilité publiques. Pour assurer la publicité de l'événement, les Big Brother Awards font l'objet d'annonces (autocollants, affiches, flyers, communiqués de presse...) et d'invitations auprès de journalistes. Par ailleurs, la totalité des argumentaires, ainsi que la composition des jurys de l'ensemble des éditions, restent accessibles au public sur le site internet de l'association. Notre lecteur pourra ainsi facilement s'y reporter (http://bigbrotherawards.eu.org).

De 2000 à 2013, les Big Brother Awards ont connu un total de 11 éditions (l'organisation de l'événement n'a pas eu lieu en 2008, en 2011 et en 2012, principalement par manque de disponibilité des membres les plus actifs du collectif). Les catégories de récompense ont pu légèrement varier au fil des années. Mais, pour l'essentiel, elles correspondent aux catégories suivantes: «Orwell Etat \& Elus », "Orwell Entreprise », «Orwell Localités », « Orwell Novlang », «Orwell Ensemble de son œuvre » et « Mention Spéciale», auxquelles s'ajoute le «Prix Voltaire». La catégorie «Orwell Novlang» est la seule qui concerne explicitement des pratiques langagières : c'est elle qui nous intéresse dans ce travail, et sur laquelle nous donnons quelques détails plus bas.

Dans l'immédiat, nous souhaitons souligner combien les prix, qu'ils portent d'ailleurs sur des objets langagiers ou non, constituent une ressource intéressante pour l'analyse du discours.

\subsection{Prix, trophées, distinctions et anti-prix : des occasions de déploiement du genre épidictique}

Ainsi que nous venons de le voir, l'organisation de distinctions représente l'activité centrale de Privacy France. Or, pour l'analyste du discours, les prix et les anti-prix constituent des terrains d'enquête 
particulièrement riches : à leur occasion, se déploient des discours caractéristiques du genre épidictique, au fil desquels le locuteur met en relief les valeurs de la communauté dont il se réclame (que cette communauté soit une profession, une spécialité artistique, une activité de loisir, un sport...). La catégorie « Orwell Novlang » étant un anti-prix relatif à des pratiques langagières, il est susceptible de donner lieu à l'expression de valeurs relatives à la langue et/ou au discours.

Le vaste domaine des prix, trophées, récompenses, distinctions et médailles, qu'augmente le développement contemporain des chartes et des labels, ainsi que des classements et des palmarès, est l'occasion pour les acteurs sociaux de produire des discours qui mettent en avant certaines valeurs (ou qui au contraire dénoncent certaines valeurs, dans le cas des anti-prix). La rhétorique aristotélicienne avait bien identifié, sous le nom de "genre épidictique ", l'existence de tels types de discours portant plus particulièrement sur les valeurs, qu'ils les promeuvent (discours d'éloge) ou bien qu'ils les condamnent (discours de blâme). Les allocutions de dépôt de gerbe, les discours de cérémonie de remise de diplômes, ou encore les discours de promotion dans l'ordre des palmes académiques, sont autant d'illustrations de la persistante fertilité des discours épidictiques, et signalent combien ceux-ci peuvent constituer des corpus privilégiés pour l'analyse du discours. Un récent travail d'Anthony Saber (2011) en fournit un exemple à travers l'étude de la citation pour faits de guerre dans l'armée américaine.

Dans la grande diversité des prix existants, une partie seulement porte sur le langage, le discours ou la langue (le sport, ou encore la gastronomie par exemple, étant sans doute mieux représentés). Dans le domaine du discours politique, qui nous intéresse plus directement, des recherches restent à mener pour identifier et analyser les prix en présence: «Prix du Livre politique» initié par l'association Lire la société, « Prix de l'humour politique » du Press Club de France, «Y'a Bon Awards » de l'association Les Indivisibles, «Les Bobards d'or » de la Fondation Polémia, «Prix de la Carpette anglaise » organisé dans un esprit de « défense de la langue française »... C'est le prix « Orwell Novlang » qui fait l'objet de notre étude, mais il est certain que chacun de ces prix mériterait une enquête détaillée. Parce qu'il s'attache à valoriser ou à dénoncer un objet langagier, chacun de ces prix suscite des discours métalinguistiques épidictiques: chacun donne lieu à l'expression de jugements plus ou moins explicites portant sur la langue et/ou le discours.

A ce titre, chacun de ces prix peut permettre de constituer un corpus qui intéresse l'étude du sentiment linguistique ordinaire. De quelles pratiques langagières chacun de ces prix fait-il l'éloge ou le blâme ? Quels usages de la langue et/ou du discours valorise-t-il ou condamne-t-il ? Quelles représentations implicites de la « bonne » langue et/ou du «bon » discours donne-t-il à voir ? En quoi peut-il être un lieu d'observation de certains éléments de «linguistique populaire», entendue, au sens le plus général, comme l'ensemble des représentations « spontanées », «naturelles » que les sujets parlants et les groupes élaborent concernant le langage, les langues, les phénomènes linguistiques, les règles de la communication (Colombat, Fournier, Puech 2010 : 57) ? Dans quelle mesure chacun de ces prix porte-t-il des appréciations sur divers types d'hétérogénéités (Authier-Revuz 1995), relevant en particulier de la non-coïncidence du discours à lui-même (jargon des autres, mots à la mode...) ou de la non-coïncidence entre les mots et les choses (usages antonymiques ou euphémisants...) ? Comment ces discours d'éloge et de blâme rejoignent-ils d'autres discours ordinaires ou savants sur la langue et/ou le discours comme « beaux » (Siouffi 2010) ou comme « vertueux » (Paveau 2013) ?... Chacune de ces questions appelle des développements à travers l'étude minutieuse des différentes récompenses se rapportant à des objets langagiers.

Par ailleurs, dans la mesure où les organisateurs de ces prix ne définissent que rarement de manière explicite l'objet langagier qu'ils célèbrent (ou condamnent), c'est souvent par l'analyse des discours prononcés lors de la cérémonie ou des documents préparatoires à la remise du prix que l'on peut approcher la conception que ces locuteurs se font de leur objet. Par exemple, le «Prix de l'humour politique » ne définit que minimalement ce qu'est l' ' humour », les «Y'a Bon Awards » ne définissent nulle part ce qu'est un «propos raciste » alors que tel est leur objet, et la documentation du prix «Les Bobards d'or » ne fournit pas de définition de ce qu'est un «bobard», ni même un «mensonge ». Cette faible activité définitionnelle, qui paraitrait fâcheuse dans un contexte scientifique académique, semble 
moins surprenante dans le cadre d'une manifestation non-savante. Dans tous les cas, le caractère très empirique de la démarche adoptée par ces locuteurs en fait l'un des intérêts pour l'étude de la linguistique populaire : il conduit l'analyste à chercher, parfois en creux, ce que les locuteurs ordinaires désignent à travers les termes métalinguistiques qui sont leur objet (" humour», " propos raciste », «bobard», « novlangue », « petite phrase », « tweet médiatique », « livre politique »...).

C'est dans le cadre de questionnement que nous venons d'évoquer que les discours produits par les militants des Big Brother Awards nous intéressent. D'une part, développant des discours à propos de pratiques langagières qu'ils blâment, ces locuteurs nous donnent à voir ce qu'est pour eux une pratique langagière condamnable. D'autre part, en estampillant ces pratiques langagières sous le sceau «Orwell Novlang ", ces locuteurs nous aident à appréhender ce qu'ils entendent par «novlangue », sous différentes graphies.

\subsection{La catégorie « Orwell Novlang » : un instrument de critique du discours}

La catégorie «Orwell Novlang » a été créée en 2004, soit à partir de la cinquième édition des Big Brother Awards. Le prix correspondant à cette catégorie a été décerné sept fois, compte tenu des interruptions de 2008, 2011 et 2012. A l'instar des autres anti-prix des Big Brother Awards, le prix « Orwell Novlang » est explicitement défini dans sa visée dénonciatrice. La documentation de Privacy France, ainsi que le paragraphe introductif du prix pour chacune des éditions, le définissent en effet selon ces termes :

Le prix «Novlang» est décerné aux opérations de propagande politique, commerciale, publicitaire, médiatique ou autres ayant pour objet, ou pour effet, d'attenter au droit à la vie privée, d'appeler à moins de libertés, de banaliser la société de surveillance, par leur manipulation du discours sécuritaire, leur stigmatisation de « menaces » opportunistes ou toute autre instrumentalisation du débat public.

La dimension langagière de l'objet concerné est assez nettement exprimée (comme en témoignent les formulations «opérations de propagande », « manipulation du discours sécuritaire », «stigmatisation de "menaces" », « instrumentalisation du débat public »), ce qui fait clairement de ce prix un instrument de critique du discours: le prix "Orwell Novlang» participe d'une démarche militante qui vise des pratiques langagières. En revanche, et sans que cela nous surprenne, la définition du prix n'indique que par fragments et en creux ce que peut désigner «Novlang». Elle ne donne pas non plus de renseignements précis sur les formes ou sur les procédés qui témoigneraient de ces mésusages du langage. L'un des objectifs de notre travail est de mieux cerner ces formes ou ces procédés, tels que conçus dans les représentations linguistiques spontanées des militants.

Lors de la cérémonie de remise des Big Brother Awards, la définition de la catégorie « Orwell Novlang » est lue pour être rappelée au public. Elle est introduite, comme c'est également le cas sur la documentation écrite, par la citation d'une phrase de George Orwell (1946: 169) tirée du célèbre article « La politique et la langue anglaise »:

"Quand l'atmosphère générale est mauvaise, le langage ne saurait rester indemne. "

Comme pour chaque prix, les organisateurs présentent ensuite rapidement les nominés auprès du public, sur la base d'un résumé de l'argumentaire les concernant. Un membre du jury est alors appelé pour ouvrir l'enveloppe contenant le nom du lauréat. Celui-ci est annoncé au public, auquel est présenté en détail le gagnant, dont l'argumentaire est lu et commenté. Comme il est d'usage pour les prix positifs, le lauréat est invité à venir chercher son certificat ainsi que le trophée. Prévenus par les organisateurs, les différents lauréats ne se sont jusqu'à présent jamais déplacés.

Le certificat, dont les formulations et la présentation ont varié selon les années, comporte notamment le nom du lauréat et le motif de son élection (par exemple à travers une formulation telle que : "Pour avoir remplacé le terme... »). Le trophée, quant à lui, est un objet d'une trentaine de centimètres de haut. Placé sur un socle et d'aspect doré, il représente une seringue plantée dans un cerveau, dans lequel cette seringue est à demi-enfoncée pour une injection. Il est intéressant d'observer que ce trophée ne renvoie pas à la langue, ni même à l'oreille et l'écoute, ou à la vue et la lecture, mais au cerveau et à la pensée. De façon très littérale, le trophée du prix «Orwell Novlang » évoque ainsi l'influence du langage (ou du discours, ou de l'usage de la langue, ou de la langue...) sur la pensée. De ce point de vue, il semble 
s'appuyer sur le paradigme de l'influence directe et massive des messages sur le public (précisément appelé dans les travaux académiques le «modèle de la seringue hypodermique»), lequel dominait les théories des effets des médias et de la propagande politique dans les années 1930-1945.

Pour identifier les pratiques langagières auxquelles le prix « Orwell Novlang » renvoie, nous avons pris appui sur des données empiriques produites par les organisateurs du prix. En effet, le corpus que nous soumettons à l'étude est constitué de la totalité des argumentaires produits par le collectif associatif pour instruire le dossier de chacun des nominés, soit au total 40 argumentaires (5 nominés en 2004, 7 en 2005, 6 en 2006, 6 en 2007, 4 en 2009, 7 en 2010, 5 en 2013). Chaque argumentaire se présente sous la forme d'un texte relativement bref et doté d'un titre (environ 3500 à 4500 signes espaces compris pour la plupart des argumentaires). Il comporte, en note, des liens hypertextes vers des sources d'information publiques (articles de presse, rapports...).

Ce corpus de 40 argumentaires constitue un corpus clos, en ce sens qu'il rassemble la totalité des textes produits par une organisation donnée dans un cadre institutionnel constant. En revanche, derrière l'unicité d'appartenance générique, ces textes peuvent présenter une certaine hétérogénéité formelle interne : ils ont été produits par des scripteurs différents (c'est-à-dire les militants qui se sont succédé dans l'organisation du prix), et n'ont pas fait l'objet d'un lissage très systématique. Du point de vue de l'étude du sentiment linguistique ordinaire, cette remarque nous conduit à souligner que les argumentaires étudiés ne forment pas nécessairement système concernant l'objet qu'ils visent : ils ne sont pas des textes qui prétendraient décrire les réalisations d'une catégorie qui aurait été définie préalablement (la «novlangue », dont les militants chercheraient à récolter des occurrences), mais plutôt des textes qui décrivent des réalisations dont intuitivement des militants estiment qu'elles relèvent d'une certaine catégorie (celle que vise le prix « Orwell Novlang »), sur laquelle porte un présupposé d'existence et un implicite définitionnel. Nous rejoignons là les observations précédentes sur le caractère empirique de la démarche que nous analysons, et qui la rend si intéressante pour l'étude des intuitions concernant la langue et le discours.

La «novlangue» est au cœur des textes produits par les militants de Privacy France pour mener la critique des discours qui témoignent de ce qu'ils appellent l'«idéologie sécuritaire ». Nous proposons donc à présent de situer l'objet nommé «novlangue », de manière à mieux saisir le substrat de la dénonciation opérée par les Big Brother Awards.

\section{La « novlangue » comme nom de langue imaginaire : de la fiction orwélienne aux naturalisations contemporaines}

\subsection{La « novlangue » dans 1984 de George Orwell : quelques rappels}

Notre lecteur aura compris que le collectif militant qui organise les Big Brother Awards présente l'ensemble de son action comme étant inspirée par le journaliste et écrivain George Orwell (1903-1950), souvent considéré comme une figure emblématique de l'opposition aux totalitarismes, en particulier dans leurs pratiques de surveillance des individus. Il aura aussi compris que le roman 1984, publié en anglais en 1949, constitue l'univers de référence des Big Brother Awards : on retrouve notamment dans les documents et la cérémonie le nom de l'entité qui exerce le pouvoir totalitaire dans le roman (Big Brother), le nom du personnage principal (Winston Smith), et le nom de l'objet langagier qui est au centre de 1984 («novlangue »).

Ici n'est pas le lieu pour décrire en détail ce qu'est la «novlangue » dans 1984. Nous nous contentons de procéder à quelques rappels, que le lecteur pourra compléter en consultant le roman lui-même, mais aussi en lisant le commentaire synthétique qu'en fait Jean-Jacques Courtine (1985), l'essai qu'y consacre Jacques Dewitte (2007), l'étude approfondie qu'en offre Sandrine Sorlin (2010), ou encore l'analyse qu'en propose Alice Krieg-Planque (2012a). 
Dans le vaste champ de ce que Marina Yaguello ([1984] 2006) a étudié sous le jour des «langues imaginaires », la «novlangue» (ou Newspeak en anglais) apparaît réellement comme une langue construite : la «novlangue » est très concrètement élaborée par les personnages de la fiction, et non pas préexistante dans l'univers diégétique. Le roman décrit en effet un monde dans lequel des acteurs sociaux travaillent à construire systématiquement une nouvelle langue (en remplacement de l'ancienne, dite «ancilangue », ou Oldspeak) et à la mettre en œuvre grâce à un appareil bureaucratique, dans la perspective d'un projet idéologique précis.

Pour le lecteur de 1984, ce qu'est la «novlangue » peut être appréhendé à travers ce qui en est dit dans le récit lui-même, mais aussi à travers l'appendice « Les principes du novlangue » qu'Orwell lui consacre en fin d'ouvrage. Du point de vue de sa fonction politique, le but de la «novlangue » est, pour reprendre les termes d'Orwell (1950: 422), "non seulement de fournir un mode d'expression aux idées générales et aux habitudes mentales des dévots de l'Angsoc [le parti unique au pouvoir], mais de rendre impossible tout autre mode de pensée ». En tant que code linguistique, la «novlangue » présente certains traits remarquables, bien explicités dans l'appendice. Globalement, elle se caractérise par l'appauvrissement du vocabulaire, et un principe d'éradiction de la synonymie et de diverses formes d'équivocité.

Par ailleurs, il semble utile d'avoir constamment à l'esprit que «novlangue » est un terme néologique choisi par les traducteurs de l'anglais vers le français pour cet autre néologisme qu'est Newspeak, forgé en anglais par Orwell : les effets de sens que peut provoquer «novlangue » ne sauraient être identiques à ceux que peut engendrer Newspeak. Concernant le genre du nom, c'est l'instabilité qui prévaut en français : si la traductrice de l'édition la plus souvent citée a choisi le masculin («le novlangue »), les usages les plus fréquents laissent au contraire prévaloir le féminin («la novlangue »), norme dominante à laquelle nous nous conformons nous-même ici.

\subsection{Les postérités du terme "novlangue »: réifications, naturalisations, effets de glossonymie}

Rien n'obligeait le terme «novlangue » à connaître la notoriété qu'il connait aujourd'hui, du moins en langue française (les usages de Newspeak en anglais, de Neusprech en allemand, de neolingua en espagnol... méritent des enquêtes spécifiques). Nombre de langues ou de parlers imaginaires, que leurs auteurs avaient pourtant pris le soin de nommer (le «quenya » de Tolkien, le «klingon » de Okrand...), ne connaissent pas une célébrité d'une telle ampleur.

Le terme «novlangue », dans le contexte de démarches critiques à l'égard du discours politique, connaît une postérité remarquable. Jaime Semprun (2005) et Alain Bihr (2007) y ont chacun consacré un essai, dans lequel ils tendent tous deux à faire de "novlangue » le nom d'un système de signes cohérent et organisé. Sur des modes moins étoffés mais tendant également à faire de la «novlangue " un objet grammaticalisable, nombreux sont les acteurs sociaux, situés préférentiellement à l'extrême droite ou bien dans les mouvances du socialisme libertaire, qui s'emploient à produire divers «dictionnaires de novlangue ", publiés sous forme d'opuscules ou édités sur internet (Krieg-Planque 2012b et 2012c). Plus globalement, fréquentes sont les critiques du discours politique qui ont recours, d'une façon ou d'une autre, à une allusion à la «novlangue » imaginée par Orwell, stigmatisant souvent de façon sectorielle la « novlangue gouvernementale », la «novlangue administrative» ou encore par exemple la «novlangue managériale ».

Chacun de ces usages de «novlangue », qu'il soit occasionnel ou plus organisé, est le témoignage de l'héritage légué par Orwell en matière de métalangage ordinaire : grâce au roman 1984, le vocabulaire métalinguistique ordinaire s'est enrichi d'un terme nouveau, témoignant de la vitalité de la réflexivité comme possibilité inhérente à la faculté de langage. Le prix «Orwell Novlang» s'inscrit dans la continuité des usages multiples que nous venons d'évoquer : il est un, parmi d'autres, des témoignages des emplois de "novlangue » comme terme métalinguistique ordinaire relatif aux codes et aux parlers. Son étude, proposée ici, entend donc nourrir le champ des recherches sur le métalangage ordinaire, lequel a fait l'objet d'un intérêt croissant depuis le milieu des années 2000, ainsi que suffit à l'illustrer la 
floraison des numéros de revue sur le sujet (Beacco dir. 2004, Achard-Bayle et Paveau dir. 2008, AchardBayle et Lecolle dir. 2009, Lecolle 2014), qui prolongent et enrichissent les travaux fondateurs menés dans une perspective linguistique et sémiotique par Josette Rey-Debove (1978) et dans une optique plus énonciative et discursive par Jacqueline Authier-Revuz (1995).

L'une des conséquences de l'origine situable du terme «novlangue » est qu'elle semble cautionner l'impression selon laquelle il existerait réellement quelque chose qui s'appellerait (la) «novlangue ». L'autorité morale que peut légitimement représenter Orwell ne peut que renforcer cette impression, Orwell apparaissant en quelque sorte comme quelqu'un qui aurait bel et bien montré la réalité d'un tel type d'objet langagier. Dans tous les cas, il semble bien que «novlangue» fonctionne, dans le vocabulaire contemporain, comme le nom d'un code, d'un parler ou d'un type de discours qui existerait de manière objectivable. Or, de notre point de vue, la «novlangue » (et nous adopterions strictement la même position à propos de la «langue de bois » ou du «politiquement correct») n'existe en rien de manière objectivable, mais existe en tant que terme métalinguistique ordinaire servant à apprécier des usages et des pratiques. Le travail du linguiste et/ou de l'analyste du discours consiste dès lors à dénaturaliser ce terme pour en décrire les emplois.

Dans le travail que nous avons consacré à la «novlangue», signalé précédemment (Krieg-Planque 2012a), nous nous sommes intéressée en particulier à « novlangue » comme nom de langue imaginaire. Nous avons remarqué que ce terme est toujours mobilisé pour qualifier l'altérité discursive : dans les discours politiques et sociaux contemporains, "novlangue » est utilisé pour désigner, négativement, le discours de l'autre. Nous avons également mis en évidence un effet de glossonymie que produit en français le terme "novlangue », et nous soulignons que cet effet participe d'une réification du discours d'autrui : le terme "novlangue » produit sur ce qu'il qualifie ou désigne des effets de réification langagière par lesquels les propriétés supposées de ce qu'est une langue (clôture, ordre...) sont transférées à ce qui, à certains égards, n'est «que» du discours produit dans une langue (la langue française, par exemple, pour ce qui concerne les propos stigmatisés par les Big Brother Awards). C'est un tel type d'analyse, mené ici sur le corpus spécifique des Big Brother Awards, que le présent travail entend poursuivre, dans le cadre plus large de nos recherches sur le métalangage ordinaire dans ses usages critiques, militants et/ou polémiques en contexte socio-politique.

\section{La « novlangue » du prix " Orwell Novlang » : des pratiques discursives diverses au service d'un même supposé dévoiement du langage}

Nous proposons à présent de cerner les usages qui, dans le sentiment linguistique spontané des militants des Big Brother Awards, renvoient à ce que ces locuteurs appellent «novlangue ». Pour cela, nous analysons les 40 argumentaires présentés plus haut: ces courts textes constituent ainsi les données empiriques depuis lesquelles nous cherchons à saisir des acceptions de ce terme métalinguistique ordinaire qu'est «novlangue » et/ou des caractérisations de ce que réalise la «novlangue », toujours d'après les Big Brother Awards.

\subsection{Un code langagier préférentiellement défini "en creux »: les rares définitions de " novlangue "}

Si l'on s'en tient aux définitions de «novlangue» qui sont explicitement formulées dans les argumentaires, on constate que ce terme est rarement défini. Quand bien même on retient une conception libérale de ce qu'est une définition, en incluant par exemple les énoncés définitoires ordinaires ou les appositions, le corpus n'offre au plus que deux opérations de définition sur l'ensemble des 40 textes considérés. Nous sommes ici témoins de la faible activité définitionnelle évoquée au début de cette contribution à propos du discours métalinguistique épidictique.

L'une des deux définitions figure dans un argumentaire de 2010. La valeur définitionnelle de l'apposition y est renforcée par les guillemets, qui produisent un effet de prélèvement dans un intertexte faisant autorité : 
Brice Hortefeux aime tellement la vidéosurveillance qu'il a décidé d'en changer le nom, au profit de la «vidéoprotection». Un parfait exemple de novlangue, «simplification de la langue destinée à rendre impossible l'expression des idées subversives et à éviter toute formulation de critique de l'État ». [brice 2010]

En la circonstance, une rapide enquête permet de retrouver la trace de ce segment apposé : elle se trouve dans la notice « novlangue » de l'encyclopédie collaborative en ligne Wikipédia.

L'autre définition, qui est à la fois ambiguë, restrictive et formellement déjà éloignée d'un énoncé définitoire canonique, figure dans l'un des argumentaires de l'édition 2005 :

Il [Nicolas Sarkozy] manie avec tact cette «novlang » contemporaine qui vise à maintenir le citoyen dans une dépendance et une soumission aux structures de l'état et des entreprises, afin de faire encore mieux accepter aux individus la présence des contrôles en tous genres et de l'inaction de toute rébellion. [sarko 2005]

Les pages qui suivent nous donneront l'occasion de revenir sur les contenus de ces définitions.

Au-delà des rares définitions identifiables, c'est à travers les usages du terme " novlangue » lui-même qu'il est possible de cerner les contours de l'objet langagier que visent les Big Brother Awards. Sur ce point, le corpus fait apparaître des caractéristiques remarquables dont nous signalons ci-après rapidement les plus saillantes. Ces observations convergent pour faire de "novlangue » un terme particulièrement plastique.

Sur l'ensemble des 40 argumentaires, on rencontre un total de 21 occurrences de «novlangue » (toutes graphies confondues) ou d'un de ses éventuels dérivés. Du strict point de vue des formes graphiques, le terme connaît une certaine malléabilité : on trouve ainsi les formes «novlangue » (13 occurrences), « Novlang » avec une majuscule en dehors de l'initiale d'une phrase (4), «novlang » (2), « Novlangue » avec une majuscule en dehors de l'initiale d'une phrase (1) et «nov'langue » (1). La forme « Novlang », qui est celle qu'avaient retenu les organisateurs à la création du prix en 2004, est tendanciellement caractéristique des premières éditions. En revanche, la forme "novlangue » devient la forme exclusive à partir de 2007, à la seule exception de la forme « Novlangue » qui est présente une fois en 2010.

La diversité des scripteurs successifs et l'absence d'homogénéisation systématique des textes étudiés, que nous avons signalées plus haut, nous mettent en garde contre des conclusions hâtives. Néanmoins, nous ne pouvons manquer d'observer que l'évolution de «Novlang » vers " novlangue » tend à privilégier la forme la plus familière en langue française: d'une part, une lettre minuscule à l'initiale d'un nom commun est plutôt la règle en français (contrairement à l'allemand, ou exceptionnellement à l'anglais s'il s'agit d'un glossonyme) ; d'autre part, " langue » est une forme préexistante en français (contrairement à « lang », qui évoque peut-être dans les représentations sur les langues une graphie de langue germanique, qu'elle soit allemande ou scandinave). Peut-être peut-on donc voir dans le passage de « Novlang» vers « novlangue » l'indice d'une appropriation du terme par la communauté des scripteurs militants, lesquels se seraient progressivement familiarisés avec un mot initialement perçu comme un xénisme. Bien des hypothèses sont imaginables, mais la nature et la taille de notre corpus incitent surtout à la prudence interprétative sur cette intéressante question de la graphie des glossonymes.

Au plan grammatical, c'est également la souplesse qui caractérise "novlangue ». Si le terme est majoritairement employé comme nom (14 fois), il est parfois employé comme adjectif ( 7 fois). Il faut souligner que cet emploi adjectival consiste alors toujours en une dérivation impropre, c'est-à-dire en un changement de classe grammaticale sans marque de dérivation suffixale. On rencontre ainsi les syntagmes «pensée novlangue », " esprit novlangue », " terme novlangue », " interprète Novlang », « discours Novlang » (ce dernier syntagme survient à 3 reprises, toujours avec majuscule). Ces énoncés avec emploi adjectival, outre qu'ils témoignent de l'adaptabilité syntaxique du terme, ont des conséquences au plan sémantique. En effet, ils contribuent à construire la grande amplitude sémantique du terme : l'adjectif «novlangue » se trouve d'autant plus ouvert sémantiquement qu'il est privé d'un morphème suffixal («-esque », «-ard/arde », «-eux/euse », «-iste »...) qui en orienterait minimalement la signification en tant qu'adjectif. 
L'usage des déterminants quand «novlangue » est employé comme nom mérite au moins qu'on le remarque. En dehors du recours, assez attendu, au déterminant défini « la » et au déterminant indéfini « une », on observe en effet deux emplois de déterminants habituellement destinés aux noms massifs, en l'occurrence l'article partitif « de la » et le déterminant complexe « un peu de » :

De la novlangue pur jus à lire sans modération. [cnil 2010]

Pour justifier cette frénésie [l'installation de caméras], rien ne vaut un peu de novlangue : pour lui la vidéosurveillance est source de «tranquillité ». [goujon 2007]

On peut bien entendu voir là de simples effets plaisants ou ludiques, en particulier dans le premier énoncé. Mais il nous semble qu'il faut également prendre les effets de sens au sérieux, et considérer avec attention la construction de «novlangue » comme nom de masse. En effet, dotée des propriétés des noms de masse, la « novlangue » apparaît comme une matière langagière qui peut participer, en s'y fondant, à des ensembles langagiers plus vastes qu'elle. Dans la version massive de «novlangue », les manifestations de ce code langagier peuvent alors être une unité lexicale à l'intérieur d'une phrase, une phrase à l'intérieur d'un discours, un paragraphe à l'intérieur d'un texte, une prise de parole à l'intérieur d'un échange, etc. Une telle représentation n'est pas sans conséquence sur la conception de ce qu'est la «novlangue » dans le sentiment linguistique spontané : cette version massive peut en effet fonder la vision de la «novlangue » comme élément de contamination d'une matière langagière plus saine. Introduite sous la forme discrète d'un fragment, la «novlangue » peut être l'ingrédient malsain à partir duquel la corruption se dissémine dans un discours juste ou dans une langue intègre. Pour les observateurs critiques du politique, la «novlangue » apparaît alors comme un dangereux - car peu visible - vecteur de dégradation des pratiques langagières.

Enfin, parmi les contextes d'emploi les plus remarquables du terme «novlangue », on ne peut manquer de relever la récurrence de formulations qui expriment une réalisation sur une échelle de graduation. En effet, on trouve dans l'environnement de «novlangue » des expressions diversifiées qui ont pour point commun d'exprimer l'excellence de la réalisation de cet objet qu'est la «novlangue », par rapport à des réalisations qui seraient moins typiques. Parmi les formulations concernées se trouvent, outre « de la novlangue pur jus » et « parfait exemple de novlangue » cités plus haut, des expressions telles que « belle novlangue politico-scientifique », «bel exemple de nov'langue marketing », "le meilleur interprète Novlang du gouvernement», ou encore « une novlangue particulièrement exemplaire ». Bien entendu, s'agissant de textes rédigés pour étayer la délivrance d'une récompense, ces énoncés sont en partie à relier à la question de la compétition et de l'évaluation : celui qui présente le meilleur exemplaire de la catégorie «novlangue » est celui qui obtiendra le trophée. Mais, s'agissant d'un anti-prix, c'est-à-dire de la stigmatisation d'une pratique jugée négativement, il faut aussi appréhender ces énoncés pour leur valeur rhétorique antiphrastique, et voir en eux le sentiment de scandale moral qui s'exprime face à des usages dévoyés du langage.

Il apparaît ainsi que si «novlangue » est rarement explicitement défini, l'observation des usages du terme en contexte permet d'approcher certaines caractéristiques du référent langagier tel que conçu par les Big Brother Awards. Conformément à un principe cher à la linguistique et à l'analyse du discours, c'est en observant les emplois d'un signe que l'on peut précisément rendre compte de sa valeur. Pour compléter cette compréhension de «novlangue », nous proposons à présent d'analyser la façon dont sont caractérisés les propos (déclarations, textes, comportements langagiers...) qui ont été nominés, autrement dit qui ont été présumés dignes de relever de la « novlangue » du point de vue des Big Brother Awards. Dans les subdivisions qui viennent, nous cherchons donc à repérer les référents - qui sont des référents langagiers, s'agissant d'un terme métalinguistique - auxquels « novlangue » renvoie.

\subsection{Des usages dominés par une intentionnalité : vouloir tromper et dissimuler}

L'intention, en tant qu'elle est de surcroit une intention trompeuse ou dissimulatrice, est une caractéristique forte des propos que condamnent les Big Brother Awards. Dans les définitions que l'association fournit des différentes catégories de prix, la simple production d'effets sans intention n'est pas exclue du blâme (par exemple, le prix « Orwell Entreprise » entend condamner les organisations qui violent la sphère privée «par profit ou simple négligence »). La catégorie «Orwell Novlang » est 
également dans ce cas. Pourtant, de fait, dans les argumentaires analysés, la présence d'une intention caractérise de façon récurrente les propos nominés pour l'anti-prix.

Parmi les diverses formulations disponibles en langue française pour exprimer la finalité («pour », « afin de », «dans le but de »...), celles qui ont recours à la préposition «pour» sont de loin les plus représentées dans le corpus. L'un des énoncés considérés semble particulièrement symptomatique de l'imaginaire linguistique culturaliste sur lequel l'idée de «novlangue » s'appuie. Cet énoncé évoque en effet une volonté d'agir sur le langage pour modifier la façon de penser. Il recourt au syntagme "pour conditionner ", et il constitue le titre de l'argumentaire du nominé et lauréat de 2004, le groupement d'industries électroniques GIXEL (il faut comprendre ainsi : «le groupement GIXEL est nominé pour discours Novlang pour conditionner...») :

Discours Novlang pour conditionner la population, surtout les enfants, aux technologies de contrôle social. [gixel 2004]

Les autres syntagmes prépositionnels en "pour + verbe» exprimant une intentionnalité sont "pour justifier » $(2$ occurrences $)$, « pour dissimuler » $(2$ occurrences $)$, et « pour convaincre » (1 occurrence) :

Pour nous convaincre des bienfaits des nanotechnologies et des microsystèmes de surveillance, la communauté urbaine de Grenoble et son prestataire Vivagora ont maquillé en «débats citoyens » de simples conférences promotionnelles. [nanoviv 2006]

La locution «en vue de » apparaît également parmi celles qui permettent d'exprimer l'intentionnalité qui serait caractéristique de la « novlangue »:

Le ministre de l'Intérieur et président de l'UMP cultive sans cesse un sens aigü du discours biaisé et trompeur en vue d'assoir son pouvoir et ses ambitions. [sarko 2005]

Enfin, la récurrence de la locution « sous couvert de» peut être soulignée. Présente à 3 reprises, cette locution met en relief la dissimulation, l'occultation, les prétextes fallacieux et les allégations mensongères qui sont, d'après le corpus, rattachées à la «novlangue » :

C'est ce qui s'appelle se faire plumer de la plus belle manière, sous couvert de «chance unique de laisser sa marque dans le monde scientifique lié à l'industrie» (sic !), « de sécuriser les grands événements sportifs » (re-sic !) et d'une hypothétique « embauche » chez EADS. [eads 2013]

Les observations menées ici à propos de «novlangue » ne sont pas sans rappeler celles que fait au sujet du « vague » Pascale Brunner (2013), qui étudie à la fois le mot et la notion. S'intéressant également au discours de locuteurs ordinaires, Pascale Brunner remarque en effet que la caractérisation d'un discours comme "vague » peut être associée à l'attribution d'une production intentionnelle de «vague », et que cette intention peut être jugée de nature dissimulatrice, au sens où le locuteur opérerait ainsi une commode protection de soi (ex. «le gouvernement préfère rester dans le vague afin de conserver un moyen de pression $»)$. Dans le corpus que nous étudions, c'est préférentiellement à travers une caractérisation comme «joli » ou comme « doux » que les propos sont jugés dissimulateurs, ce qui les rapproche plus clairement encore d'une logique euphémisante sur laquelle nous reviendrons plus loin.

\subsection{Des pratiques de substitution : remplacer le « bon mot " par un « mauvais mot »}

Parmi les usages du langage qui font l'objet d'une dénonciation de la part des Big Brother Awards, les pratiques de substitution d'un terme par un autre sont particulièrement remarquables. Par «pratique de substitution ", nous entendons le remplacement d'un terme par un autre, au sens très matériel d'une biffure de l'occurrence d'un terme et de son remplacement par un autre terme. Dans le cas présent, le terme biffé est jugé adéquat au référent par les Big Brother Awards, tandis que le terme remplaçant est jugé inadéquat au référent. Il s'agit donc, pour les Big Brother Awards, de critiquer des locuteurs qui se livrent à l'éradication d'un «bon mot», porteur d'un juste point de vue sur le réel, et qui lui substituent un «mauvais mot», porteur d'une conception biaisée de la réalité.

Quantitativement, de telles pratiques sont assez peu fréquemment épinglées dans le corpus (3 argumentaires sont concernés sur les 40). Néanmoins elles semblent particulièrement symptomatiques des usages dévoyés du langage tels que les visent les Big Brother Awards. Elles font écho de manière 
particulièrement visible à ce que «novlangue » représente pour ce collectif associatif, ainsi qu'on le relève ci-dessous ([loi 2010]) par la référence au roman 1984.

L'un des argumentaires qui pointe cet usage a déjà été évoqué précédemment à propos de la définition de «novlangue ». Le texte justifie la nomination de Brice Hortefeux, ministre de l'intérieur, qui est ainsi caractérisé :

Adepte de la novlangue, il propose, dans la LOPPSI [Loi d'Orientation et de Programmation pour la Performance de la Sécurité Intérieure], d'effacer le terme de vidéosurveillance dans tous les textes officiels, pour y substituer celui de «vidéoprotection ». [brice 2010]

Un second argumentaire, rédigé également pour l'édition 2010, fait référence à la même thématique, en fustigeant plus précisément un rapport du député Eric Ciotti, dont il cite un extrait que voici : "Remplacement du terme "vidéosurveillance " par " vidéoprotection ». - Cet article est issu d'un amendement du Gouvernement. Dans les articles 17 et 18, celui-ci a choisi de remplacer la dénomination de "vidéosurveillance» par celle de "vidéoprotection ». En conséquence, il est nécessaire de procéder à ce changement sémantique dans l'ensemble des textes législatifs et réglementaires, ce à quoi procède cet article. » [loi 2010]

Le paragraphe introductif de l'argumentaire inscrit cette pratique de réécriture dans la continuité des pratiques décrites par George Orwell dans le roman 1984 :

Comme Winston Smith, le héros du roman « Nineteen eighty four »d'Orwell, les scribes de l'état vont devoir modifier (a posteriori) les journaux du Parti pour ne pas contredire les vérités du jour... ainsi la Surveillance devient Protection. Merci MM. Hortefeux et Ciotti. [loi 2010]

Comme on le comprend, les militants condamnent ici des pratiques qui relèvent selon eux du révisionnisme historique et de la falsification : en renommant systématiquement les objets selon le point de vue qui leur convient, les promoteurs de la «société de surveillance » contribuent à construire une perception du monde qui soit au service de leurs valeurs.

On notera que, tel qu'il est représenté par les Big Brother Awards, ce travail de réécriture est outillé par des instruments métalinguistiques à caractère normatif: l'argumentaire [loi 2010] suggère ainsi l'existence d'un dictionnaire à l'usage des révisionnistes (l'argumentaire estime que « Le dictionnaire officiel de la République va pour la première fois être modifié par la loi de manière à... »), et il est illustré d'une petite vignette qui parodie la célèbre couverture du Petit Robert, rebaptisé par allusion au président Nicolas Sarkozy Le Petit Nicolas. Le dictionnaire officiel de la Novlangue sécuritaire. Les pratiques de substitution menées par les pouvoirs que dénoncent les Big Brother Awards apparaissent ainsi non pas comme des actions plus ou moins hasardeuses occasionnées par les circonstances, mais comme des tâches conduites de manière organisée à des fins de normalisation et d'homogénéisation du vocabulaire. Les acteurs du monde sécuritaire conduiraient ainsi un travail systématique d'imposition du lexique autorisé, renvoyant du même coup dans la résistance ou la subversion les acteurs sociaux qui, voulant maintenir le lexique interdit, s'opposeraient à ces opérations de commutation falsificatrices.

\subsection{Des recours à la néologie de sens : renverser les valeurs}

Les militants des Big Brother Awards ne sont pas insensibles à la néologie de forme, comme en témoigne par exemple leur attention au surgissement du terme "vidéoverbalisation », considéré dans un argumentaire comme « un néologisme qui vient rejoindre la vidéoprotection » [videov 2013]. Aussi bien que les lexicologues du monde savant, ces militants ont l'intuition que la productivité d'un morphème est souvent socialement signifiante. Mais ces militants sont au moins aussi attentifs à la néologie de sens, dont ils pressentent qu'elle peut participer, sur un mode particulièrement insidieux et pervers, à un renversement des valeurs.

Telle qu'elle est mise en évidence dans le corpus, la néologie de sens peut évoquer jusqu'à un certain point la pratique précédemment signalée. Mais elle diffère par l'opération sémiotique que, fondamentalement, elle réalise : il ne s'agit pas de remplacer un signe par un autre signe, mais 
d'employer un signe en association avec un trait de signification qui n'y est pas habituellement présent (c'est par exemple ce qu'exprime un sentiment linguistique spontané assez communément partagé quand on parle de «plan de sauvegarde de l'emploi » pour désigner des licenciements). Le trait de signification inattendu peut éventuellement présenter une valeur antonymique, mais ce n'est pas toujours le cas.

L'un des argumentaires de l'édition 2013 porte sur les services municipaux dits de «tranquillité publique », qui ont fait leur apparition depuis quelques années dans certaines municipalités françaises. De façon détaillée, le texte expose les acceptions successives de l'expression, en soulignant combien «tranquillité publique» est historiquement associé au bon ordre et à la sécurité. Puis ce même argumentaire relève un premier usage inhabituel de l'expression en 2006 ( «En 2006, c'est Villepin [alors Premier ministre] qui la sort de son anonymat en appelant à la constitution d'une "police de tranquillité publique". »), pour s'offusquer d'un usage à contre-emploi plus généralisé dans la période récente :

[Certains maires] utilisent la tranquillité publique à toutes les sauces, pour désigner le maintien de l'ordre par les forces de police. Un néologisme paisible qui dissimule des réalités policières parfois violemment contradictoires : très récemment, le 3 juin 2013, un agent de tranquillité publique était accusé d'avoir malmené un journaliste dans la ville de Montreuil, un comble pour un garant de la « tranquillité »... [tranquil 2013]

L'argumentaire du lauréat ex-aequo 2009 présente le projet Humabio, un projet européen qui développe des techniques biométriques d'identification des personnes basées notamment sur la démarche (corpulence, vitesse de déplacement...). Le texte souligne que l'une des conséquences de ces techniques est qu'elles permettent d'identifier la personne sans contact et à distance, donc à son insu. Les Big Brother Awards relèvent alors que cette méconnaissance par la personne du contrôle qu'elle subit est fallacieusement présentée comme un avantage pour la personne contrôlée (c'est pour elle " une liberté », selon les termes du projet Humabio), puisqu'elle n'est pas entravée ou ralentie dans ses déplacements. Reprenant par ailleurs à son compte l'imaginaire linguistique culturaliste d'un « conditionnement » par le langage, l'argumentaire conclut sur la sémantique inattendue du terme «liberté » dans les discours du projet Humabio :

Le conditionnement consiste à présenter comme une «facilité » le fait d'être identifié à son insu, de manière passive. (...) Appeler « liberté » le fait de se faire voir sans le savoir, ça c'est de l'esprit novlangue à faire rougir George Orwell ! [huma 2009]

L'un des nominés de l'édition 2007, le maire de Cannes, Bernard Brochand, se voit également accusé d'avoir recours à la néologie de sens. L'objet de la critique des Big Brother Awards est une affiche de la municipalité qui valorise la présence de caméras de surveillance sur le territoire de la commune au moyen de ce slogan : «Souriez! Vous êtes protégés. » Du point de vue des Big Brother Awards, une caméra postée dans l'espace public est l'élément d'un dispositif de surveillance individuelle et de contrôle social bien plus qu'elle ne constitue un facteur de protection. Le recours au verbe "protéger » pour signifier « surveiller » apparaît ainsi comme un renversement des valeurs. Une telle appréciation repose en partie sur une conception orthonymique du langage, dont on trouve trace dans l'argumentaire à travers l'expression « discours déviant »:

L'ancien publicitaire [B. Brochand] n'a pas seulement multiplié les caméras de surveillance dans sa commune, il a surtout usé d'un discours déviant parlant de «protection » et non de « surveillance » pour justifier sa politique. [cannes 2007]

Les trois exemples qui précèdent rappellent profondément le célèbre slogan de l'Angsoc, le parti unique au pouvoir dans le roman 1984 [Orwell 1950] :

La guerre c'est la paix. La liberté c'est l'esclavage. L'ignorance c'est la force.

Ici, les pratiques langagières dénoncées par les Big Brother Awards pourraient être mises en scène de façon synthétique à travers des énoncés tels que : «La tranquillité publique c'est la violence policière et l'insécurité », « La liberté c'est le contrôle », « La protection c'est la surveillance ».

Dans ces condamnations de l'usage perverti des mots, se manifeste de façon particulièrement nette l'appui que constitue, pour une critique du discours politique, une conception orthonymique du langage : 
celle-ci, supposant qu'il existe des usages justes (au double sens d'un usage exact et d'un usage vertueux), justifie à la fois intellectuellement et moralement les initiatives qui visent à dénoncer la déviance, ainsi que s’y emploient les Big Brother Awards à travers le prix « Orwell Novlang ».

\subsection{Une logique d'assujettissement par l'euphémisation : inhiber le sens critique}

Dans le corpus, plusieurs énoncés indiquent que la caractéristique des propos épinglés par les Big Brother Awards est que ces propos sont «doux», «paisibles », «jolis », «agréables »... Parmi les 5 argumentaires comportant des qualifications de cet ordre, on peut signaler par exemple la qualification de « joli vocable » appliquée au syntagme « citoyens relais » (" sous le joli vocable de "citoyens relais" »), lequel désigne un dispositif de surveillance du voisinage dont l'argumentaire estime par ailleurs qu'il s'agit en réalité d'un « jeu de délation-surveillance » entre concitoyens [voisins 2010]. Ou encore, on peut citer cet argumentaire qui concerne un système de vidéosurveillance des déplacements des personnes âgées :

Sous le doux nom de «Protect Angel », cet appareil détecte automatiquement chaque mouvement, chaque franchissement de limite d'une zone définie au sein d'un périmètre prédéfini, présenté ici comme « lieu de vie habituel ». [geronto 2013]

De telles appréciations évoquent les propriétés de l'euphémisme. En effet, l'idée selon laquelle un mot présente à quelque égard une dimension plus « douce » que la réalité qu'il nomme est bien l'idée centrale qui préside à l'euphémisme comme type d'appréciation du rapport entre le mot et la chose : relativement à la chose qu'il nomme, le mot qualifié d'euphémisme est jugé « faible », « doux », « atténué », « fade »... c'est-à-dire en quelque sorte « en dessous » du réel à nommer (par différence avec des mots qui nommeraient « juste », et par opposition à des mots qui nommeraient « fort », « trop fort », seraient «excessifs »...). Même si le mot «euphémisme» lui-même est absent du corpus (de même que « euphémique », « euphémiser »...), nous pouvons estimer que ce qui est visé, dans les argumentaires des Big Brother Awards concernés, c'est un usage euphémisant du langage.

Comme nous l'avons remarqué dans un travail précisément consacré au «jugement d'euphémisation » (Krieg-Planque 2004), un euphémisme est toujours un euphémisme "pour quelqu'un»: par exemple, «mondialisation » n'est pas un euphémisme en soi, mais il peut l'être pour un certain type de locuteur (par exemple pour un locuteur qui y voit la dissimulation de ce qui s'appelle en réalité selon lui l'« impérialisme américain »). Dans la mesure où il exprime un jugement sur l'adéquation du mot à la chose, le locuteur qui porte un jugement d'euphémisation opère par la même occasion une évaluation axiologique. Ceci fait de l'euphémisme une catégorie particulièrement mobilisable pour la critique du discours politique et pour l'engagement en discours, ce pourquoi il avait attiré notre attention lors de la recherche publiée en 2004, dans le cadre d'un numéro de revue consacré à la prise de position en discours : à travers le jugement d'euphémisation, il s'agit pour le locuteur de jauger un terme (et en général de jauger un autre locuteur en tant qu'il emploie un certain terme) en tant qu'il nomme insuffisamment le réel.

Dans le contexte de la critique politique qui est notre objet d'étude, il est clair que l'adoucissement identifié par les militants n'est pas relevé pour ses vertus relationnelles (politesse, urbanité, prévenance...), ce qui est l'une des fonctionnalités possibles de l'euphémisme, mais pour sa capacité à inhiber le sens critique : les locuteurs que réprouvent les Big Brother Awards nomment en quelque sorte « en dessous » du réel de manière à ne pas susciter le désaccord, alors que la réalité de leurs actions mériterait qu'on leur oppose au moins la vigilance. L'usage de la préposition «sous » (dans les formulations que nous avons rencontrées : « sous le joli vocable », « sous le doux nom »...) renforce cette accusation d'une intention dissimulatrice: les acteurs du monde sécuritaire opèrent des manœuvres condamnables en s'abritant sous de «jolis mots» dont ces acteurs espèrent qu'ils les rendront irrepérables. Le jugement d'euphémisation porté par les Big Brother Awards accompagne ainsi la condamnation d'un discours qui chercherait à ne pas éveiller la contradiction. De la sorte, il apparaît comme l'un des instruments possibles de la mise en cause des discours d'autorité (Monte et Oger à paraître), aux côtés de différents genres et dispositifs de subversion de l'autorité du discours. 
Dans un argumentaire de 2007, la qualification du mot "vidéotranquillité » comme étant un «terme soporifique » [goujon 2007] nous semble relever du même type de jugement, de même que l'ensemble des appréciations qui portent sur l'usage d'un mot comme étant un usage "pour ne pas faire peur » ou " pour rassurer ». Ce registre de la peur qu'il faut bien se garder de susciter est présent par exemple dans l'argumentaire du lauréat de 2013, le service Orange Préférences, dont les Big Brother Awards estiment qu'il met en place une "sémantique rassurante [qui] rend acceptable une intrusion directe » dans la vie privée :

\footnotetext{
Dire que la navigation est «analysée » et qu'il ne s'agit que des «adresses des sites web » fait moins peur que dire aux abonnés qu'ils sont suivis à la trace $24 \mathrm{~h} / 24 \mathrm{~h}$, qu'ils naviguent sous surveillance et qu'Orange saura sur quels sites ils vont, ce qu'ils lisent, les programmes et vidéos qu'ils regardent, etc. [orange 2013]
}

Se rendre agréable pour ne pas éveiller les soupçons, s'exprimer avec douceur de manière à ne pas effrayer, utiliser des mots rassurants afin de désamorcer les critiques... telles semblent bien être, du point de vue des Big Brother Awards, les caractéristiques du locuteur de «novlangue ». Cette dernière apparaît, dans le sentiment linguistique ordinaire tel qu'étudié ici, comme un outil au service d'une inhibition du sens critique. L'adjectif «soporifique », utilisé dans l'un des argumentaires, doit être pris au sérieux : ce que les militants stigmatisent, c'est réellement l'usage du langage aux fins d'endormir la conscience politique. Le terme «assujettissement », que nous choisissons d'utiliser, ne semble pas disproportionné pour qualifier la logique à laquelle l'euphémisation obéit, du point de vue des militants des Big Brother Awards : dans la mesure où, dans une perspective foucaldienne, la mise en place d'une société de surveillance réalise réellement pour ces militants une «idéologie sécuritaire », selon l'expression utilisée notamment dans le communiqué de presse de l'édition 2013, l'usage de la «novlangue » représente bel et bien dans l'intuition de ces militants un instrument de domination idéologique.

\section{Etudier les instruments et les termes de la critique du langage politique : un objet pour l'analyse du discours}

$\mathrm{Au}$ terme de l'étude que nous venons d'exposer, deux perspectives complémentaires se dessinent pour l'analyse de la critique du langage politique.

\subsection{Genres, instruments et dispositifs : la production de la critique}

D'une part, nous avons spécifiquement porté intérêt ici aux prix et aux anti-prix, dont nous n'avons pas manqué de souligner la pertinence pour l'analyse du discours. Néanmoins, il est bien évident que l'activité réflexive qui consiste à s'emparer des pratiques langagières pour en mener la critique est susceptible de s'exercer à travers bien d'autres modes d'expression encore. Une première perspective peut ainsi concerner les instruments, les genres et les dispositifs par lesquels les locuteurs exposent leurs appréciations.

Les dictionnaires politiques, et les débats sur les « abus de mots » qui les accompagnent historiquement, ont déjà fait l'objet d'investigations, pour la période révolutionnaire qui en est fondatrice (Steuckardt 2001) aussi bien que pour la période immédiatement contemporaine (Krieg-Planque, 2012b, 2012c et 2013). La veine des dictionnaires politiques est toujours vivace, et elle mérite encore d'autres explorations.

L'écriture des diaristes, en tant qu'elle peut prendre pour objet le langage, a suscité quelques enquêtes. C'est le cas en particulier du journal rédigé de 1933 à 1945 par Victor Klemperer (1995), qui dans une démarche doublement autobiographique et testimoniale prend pour objet les transformations de la langue allemande sous emprise nazie, et observe l'apparition d'une «LTI », ou «langue du Troisième Reich » (Aubry et Turpin dir. 2012). D'autres journaux intimes et diverses écritures de soi pourraient être travaillés sous l'angle de la critique du langage.

Mais d'autres genres et instruments restent encore entièrement à explorer en tant qu'il s'y exerce une activité métalinguistique ordinaire à visée critique. Nous pouvons évoquer par exemple les cartographies lexicales, telles que celles-ci sont techniquement permises par la visualisation des données textuelles et 
telles qu'elles peuvent faire l'objet de publicisations sur internet. Ou encore, nous pensons à des initiatives émancipatrices tels que les «Ateliers de désintoxication du langage » mis en place par la coopérative d'éducation populaire Le Pavé : il s'agit, sous forme d'ateliers animés par un membre de la coopérative, d'aider les participants à apprendre à déconstruire les discours qu'ils vivent comme des modes d'oppression idéologique, dans un cadre professionnel notamment.

\subsection{Termes métalinguistiques ordinaires: vers des interrogations sur « langage », " langue », « discours », « parole »}

D'autre part, nous avons circonscrit notre enquête au terme «novlangue ». Mais il ne s'agit là que d'un terme parmi tous ceux qui permettent aux locuteurs ordinaires de parler du discours politique, pour le nommer, le décrire, le qualifier, le dénoncer, s'en plaindre, en faire l'éloge... Une seconde perspective peut ainsi concerner les termes métalinguistiques ordinaires se rapportant aux discours politiques et institutionnels.

Certains de ces termes semblent plutôt renvoyer à des codes et à des parlers : outre « novlangue », citons « langue de bois » (étudié par Sériot dès 1986), «LTI» ou «Lingua Tertii Imperii » tel que conçu par Victor Klemperer (étudié par Savatovsky 2004), «LQR » ou «Lingua Quintæ Respublicæ » tel que posé par Eric Hazan (2006), « politiquement correct» (Savatovsky 2011), « langage des Français de tous les jours », «jargon des technocrates de Bruxelles »... D'autres termes renvoient sans doute plutôt à des pratiques discursives en situation: «petite phrase» (Krieg-Planque 2011), « dérapage », « gaffe », « lapsus », « fuite », " off »... Certaines expressions engagent des conceptions de la parole publique : « retrouver sa liberté de parole », « s'exprimer sans tabou », «parler en homme libre »... Chacun de ces termes et expressions, et l'idée même qu'ils renvoient à telle ou telle dimension de l'exercice du langage, méritent des études précises en contexte. Des expressions telles que «parler vrai» ou «langage de vérité », par exemple, ne peuvent pas être situées en l'absence d'études sur corpus approfondies.

Comme l'ont suggéré quelques passages de notre étude, et comme le laisse sans doute imaginer la simple énumération des expressions ci-dessus, une enquête sur les termes métalinguistiques ordinaires se rapportant aux discours politiques et institutionnels ne peut manquer de croiser des questions essentielles pour la linguistique. Notamment, la question du découpage des frontières entre « langage », « langue », « discours » et « parole » (et, en outre, la question de ces frontières dans différentes langues), qui se pose au linguiste, surgit également de l'observation des discours ordinaires. Que désigne le mot « discours » dans l'expression «discours sécuritaire» telle qu'elle figure dans la définition du prix «Orwell Novlang »? Quelle est la conception de la «norme » qui est sous-tendue par l'expression "discours déviant » telle qu'elle est utilisée à propos des pratiques du maire de Cannes ? A quelle «langue » renvoie Le dictionnaire officiel de la Novlangue sécuritaire dont l'existence est imaginée suite à l'opération de remplacement de « vidéosurveillance » par «vidéoprotection »? De même, quelle est la « langue » de « langue de bois », celle de « LTI », celle de « LQR » ?... L'étude de la critique du langage politique amène alors à interroger, sous l'angle du sentiment linguistique ordinaire, la façon dont la discipline travaille ses objets.

\section{Corpus}

Le corpus proprement dit est constitué des argumentaires préparés par le collectif militant des Big Brother Awards en vue de la remise du prix «Orwell Novlang» pour les sept éditions concernées, soit au total 40 argumentaires analysés, qui correspondent à autant de nominés pour la période (5 nominés en 2004, 7 en 2005, 6 en 2006, 6 en 2007, 4 en 2009, 7 en 2010, 5 en 2013).

Les argumentaires cités dans le présent travail sont référencés comme suit (le titre en italique est celui donné par les Big Brother Awards) :

[gixel 2004] GIXEL (Groupement des industries électroniques) lauréat 2004

[sarko 2005] Ministre de l'Intérieur Nicolas Sarkozy nominé 2005

[nanoviv 2006] Grenoble-Alpes-Métropole (débats « Nanoviv ») nominé 2006

[cannes 2007] Bernard Brochand, maire de Cannes nominé 2007

[goujon 2007] La « vidéotranquillité » du député Philippe Goujon nominé 2007 
[huma 2009] Projet européen Humabio lauréat ex-æquo 2009

[brice 2010] Brice Hortefeux, ministre de l'intérieur lauréat 2010

[cnil 2010] La novlangue d'Alex Türk, président de la CNIL nominé 2010

[loi 2010] La « vidéoprotection » imposée par la loi nominé 2010

[voisins 2010] Les " voisins vigilants » nominé 2010

[eads 2013] EADS Innovation Works lauréat 2013

[geronto 2013] Les " gérontechnologies » nominé 2012

[orange 2013] Orange Préférences lauréat 2013

[tranquil 2013] Les services municipaux de Tranquillité Publique nominé 2013

[videov 2013] La « vidéoverbalisation » nominé 2013

Les argumentaires analysés mais qui ne sont pas cités dans la présente contribution ne sont pas référencés ci-dessus. L'ensemble des 40 argumentaires est disponible en ligne à partir de l'adresse http://bigbrotherawards.eu.org, consulté le 11 mars 2014

A ces données empiriques étudiées systématiquement, s'ajoutent les ressources documentaires, ouvrages et articles suivants, également considérés ici comme du corpus :

Big Brother Awards (2008), Les surveillants surveillés, Paris : La Découverte / Zones.

Bihr A. (2007), La Novlangue néolibérale. La rhétorique du fétichisme capitaliste, Lausanne : Éditions Page Deux.

Hazan E. (2006), LQR. La propagande au quotidien, Paris : Liber Editions, Raisons d'agir.

Klemperer V. (2000 [1995 $1^{\text {ère }}$ éd. allemand]), Je veux témoigner jusqu'au bout. Journal 1941-1945, Paris : Seuil.

Klemperer V. (2000 [1995 $1^{\text {ère }}$ éd. allemand]), Mes soldats de papier. Journal 1933-1941, Paris : Seuil.

Orwell G. (1946), «La politique et la langue anglaise », repris dans Orwell G., 2001, Essais, articles, lettres (volumes 1 à 4), Ivrea - Éditions de l'Encyclopédie des nuisances. Volume 4, 158-173.

Orwell G. (1950), 1984, Paris : Gallimard, traduit de l'anglais par Amélie Audiberti (1 ${ }^{\text {re }}$ éd. anglais 1949).

Privacy France, site web de l'association : http://bigbrotherawards.eu.org, consulté le 11 mars 2014.

Semprun J. (2005), Défense et illustration de la novlangue française, Paris: Éditions de l'Encyclopédie des nuisances.

\section{Références bibliographiques}

Authier-Revuz, J. (1995), Ces mots qui ne vont pas de soi. Boucles réflexives et non-coïncidences du dire, Paris : Larousse, 2 tomes.

Achard-Bayle, G. et Lecolle, M. dir. (2009), "Sentiment linguistique. Discours spontanés sur le lexique », Recherches linguistiques, Metz : Université Paul Verlaine, $\mathrm{n}^{\circ} 30$.

Achard-Bayle, G. et Paveau, M.-A. dir. (2008), «Linguistique populaire ?», Pratiques. Linguistique, littérature, didactique, Metz: Cresef, $\mathrm{n}^{\circ} 139-140$.

Aubry, L. et Turpin, B. dir. (2012), Victor Klemperer. Repenser le langage totalitaire, Paris : CNRS Editions.

Beacco, J.-C. dir. (2004), «Représentations métalinguistiques ordinaires et discours », Langages, Paris : Larousse, $\mathrm{n}^{\circ} 154$.

Brunner, P. (2013), «"C'est une réponse vague”. Quand le locuteur ordinaire porte des jugements sur le vague », Le Discours et la langue. Revue de linguistique française et d'analyse du discours, Université Libre de Bruxelles, $\mathrm{n}^{\circ} 4.2,175-190$.

Colombat, B., Fournier, J.-M., Puech C. (2010), Histoire des idées sur le langage et les langues, Paris : Klincksieck.

Courtine, J.-J. (1985), «La meilleure des langues : la Novlangue d'Orwell», dans Auroux, S., Chevalier, J.-C., Jacques-Chaquin, N. \& Marchello-Nizia, C. éds, La Linguistique fantastique, Paris : Denoël, 205-210.

Dewitte, J. (2007), Le pouvoir de la langue et la liberté de l'esprit. Essai sur la résistance au langage totalitaire, Paris : Editions Michalon.

Jakobson, R. (1963), Essais de linguistique générale. 1. Les fonctions du langage, Paris : Minuit.

Krieg-Planque, A. (2004), «Souligner l'euphémisme: opération savante ou acte d'engagement? Analyse du "jugement d'euphémisation" dans le discours politique ", Semen. Revue de sémio-linguistique des textes et discours, Besançon : Presses Universitaires de Franche-Comté, $n^{\circ} 17,59-79$.

Krieg-Planque, A. (2011), «Les "petites phrases" : un objet pour l'analyse des discours politiques et médiatiques », Communication \& Langages, Paris : Editions Necplus, ${ }^{\circ} 168,23-41$.

Krieg-Planque, A. (2012a), "La "novlangue" : une langue imaginaire au service de la critique du "discours autre" ", dans Branca-Rosoff, S. et al., L'hétérogène à l'œuvre dans la langue et les discours. Hommage à Jacqueline Authier-Revuz, Limoges : Editions Lambert-Lucas, 69-83. 
Krieg-Planque, A. (2012b), «Dictionnaires, glossaires et lexiques militants : pratiques profanes de la critique du langage politique », dans Aubry, L. et Turpin, B. dir., Victor Klemperer. Repenser le langage totalitaire, Paris : CNRS Editions, 299-313.

Krieg-Planque, A. (2012c), «Un dictionnaire de combat : le "Petit glossaire de la guerre civile yougoslave" comme mode d'intervention dans un espace public en crise ", Semen. Revue de sémio-linguistique des textes et discours, Besançon : Presses Universitaires de Franche-Comté, n 34, 159-171.

Krieg-Planque, A. (2013), "Le traitement du "figement" par des locuteurs ordinaires : le sentiment linguistique d' "expression toute faite" dans des contextes de critique du discours politique ", Pratiques. Linguistique, littérature, didactique, Metz-Nancy : Université de Lorraine, CRESEF, n 159-160, 189-203.

Lecolle, M. dir. (2014), "Métalangage et expression du sentiment linguistique "profane" ", Le Discours et la langue. Revue de linguistique française et d'analyse du discours, Université Libre de Bruxelles, $\mathrm{n}^{\circ} 6.1$.

Monte, M. et Oger, C. dir. (à paraître en 2015), «Discours d'autorité : des discours sans éclat(s) ? », Mots. Les langages du politique, Lyon : ENS Editions.

Paveau, M.-A. (2013), Langage et morale. Une éthique des vertus discursives, Limoges : Lambert-Lucas.

Rey-Debove, J. (1978), Le métalangage. Etude linguistique du discours sur le langage, Paris : Le Robert.

Saber, A. (2011), "Une stylistique de l'éloge : la citation pour faits de guerre dans l'armée américaine », Etudes de Stylistique Anglaise, Nanterre : Société de Stylistique Anglaise, $\mathrm{n}^{\circ} 35$.

Savatovsky, D. (2004), «Philologie d'une nov-langue, la LTI de Victor Klemperer », dans Sériot, P. et TabouretKeller, A. éds, Cahiers de l'ILSL, n 17 (« Le discours sur la langue dans les régimes autoritaires »), 205-218.

Savatovsky, D. (2011), «Le politiquement correct : un nominalisme paradoxal », dans Branca-Rosoff, S. et al. éds, Langue commune et changements de normes, Paris : Honoré Champion, 93-109.

Sériot, P. (1986), «Langue et langue de bois en Pologne », Mots. Mots, ordinateurs, textes, sociétés, Paris : Presses de la Fondation Nationale des Sciences Politiques, n 13, 181-189.

Siouffi, G. (2010), Le Génie de la langue française. Étude sur les structures imaginaires de la description linguistique à l'Age classique, Paris : Honoré Champion.

Sorlin, S. (2010), La Défamiliarisation linguistique dans le roman anglais contemporain, Montpellier: Presses Universitaires de la Méditerranée.

Steuckardt, A. (2001), «L'abus des mots, des Lumières à la Révolution », dans Siouffi, G. et Steuckardt, A. dir., La norme lexicale, Montpellier : Publications de l'Université de Paul Valéry, 177-196.

Yaguello, M. ([1984] 2006), Les Langues imaginaires. Mythes, utopies, fantasmes, chimères et fictions linguistiques, Paris : Seuil. 